\title{
Virulence genes and antimicrobial resistance pattern in Proteus mirabilis strains isolated from patients attended with urinary infections \\ to Tertiary Hospitals, in Iran
}

\author{
Azardokht Tabatabaei ${ }^{1}$, Khadijeh Ahmadi ${ }^{2}$, Alireza Namazi shabestari ${ }^{3}$, Nastaran Khosravi $^{4}$, Ali Badamchi ${ }^{5}$
}

1. Research Center of Pediatric Infectious Diseases, Rasool Akram Hospital, Iran University of Medical Sciences, Tehran, Iran.

2. Infectious and Tropical Diseases Research Center, Hormozgan Health Institute, Hormozgan University of Medical Sciences, Bandar Abbas, Iran.

3. Department of Geriatric Medicine, School of Medicine, Tehran University of Medical Sciences, Tehran, Iran.

4. Research Center of Pediatric Infectious Diseases, Rasool Akram Hospital, Iran University of Medical Sciences, Tehran, Iran.

5. Children's Medical Center hospital, Tehran University of Medical Sciences, Tehran, Iran.

\begin{abstract}
Background: Proteus mirabilis is a frequent reason for catheter-associated urinary tract infections (UTIs). The aim of this study was to identify virulence genes and antimicrobial resistance patterns in P. mirabilis strains isolated from patients who attended a tertiary hospital in Iran.

Methods: In this study, 100 P. mirabilis strains from urine samples were isolated. These isolated strains were identified by biochemical and PCR-based tests, and their antibiotic resistance was profiled through a standard procedure using 14 antibiotics. PCR assays were used to detect virulence-related genes in P. mirabilis strains. The biofilm formation of each P. mirabilis strain was examined.

Results: Of the 100 P. mirabilis isolates, 16 (16\%) were multidrug-resistant. High resistance was observed against cotrimoxazole (97\%), nalidixic acid (93\%), cefotaxime (77\%), and amoxicillin (62\%). Sixty of the 100 isolates showed resistance against extended-spectrum cephalosporins. The prevalence rates of the genes related to the virulence factors in this study were $\mathrm{mrpH}(100 \%)$, ucaA (91\%), hpmA (94\%), zapA (95\%), ptaA (100\%), ureG (100\%), pmfA (100\%), fliC (97\%), and mrpA (90\%) using PCR method. Strong biofilm formation was observed in 20\% (5/25) of the strains isolated from non-catheterized samples and $80 \%(20 / 25)$ of strains isolated from catheterized samples.

Conclusions: Resistance to antibiotics and the prevalence of pathogenicity genes are high in Proteus mirabilis strains iolated from UTIs.

Keywords: Antibiotic resistance, Proteus mirabilis, biofilm, virulence factors.

DOI: https:// dx.doi.org/10.4314/abs.v21i4.22

Cite as: Tabatabaei A, Abmadi K, Shabestari AN, Khosravi N, Badamchi A. Virulence genes and antimicrobial resistance pattern in Proteus mirabilis strains isolated from patients attended with urinary infections to Tertiary Hospitals, in Iran. Afri Health Sci. 2021;21(4):167784. bttps:// dx.doi.org/10.4314/ahs.v21i4.22
\end{abstract}

\section{Background}

Indwelling urinary catheters are the most commonly used medical devices and are employed in a wide range

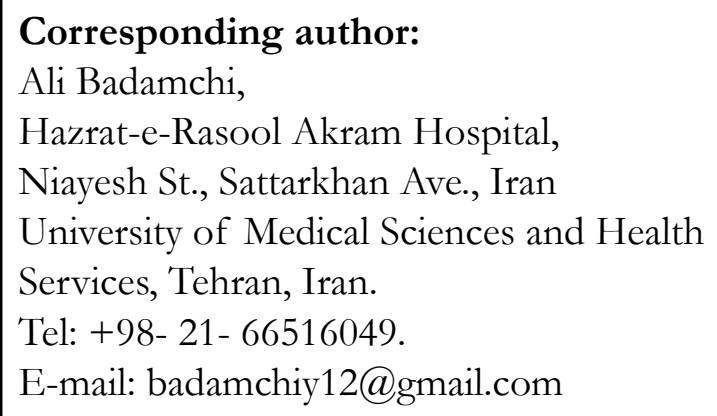

of bladder management regimens in hospitals, community care settings, and nursing homes ${ }^{1}$. It has been estimated that millions of urethral catheters are used each year, with many applied for long-term bladder control in public or nursing home settings ${ }^{2}$.

P. mirabilis is investigated as a frequent reason for catheter-associated urinary tract infections (UTIs), which can be caused by urolithiasis, and the development of bladder or kidney stones arises from the alkalinization of urine from urease-catalyzed urea hydrolysis ${ }^{3}$. The bacterium is Gram-negative and rod-shaped and is recognized by its swarming activity. 
Swimming motility may facilitate contact with uroepithelial cells, thereby promoting internalization and cytotoxicity ${ }^{4}$. Swarm cells, in particular, have been postulated to contribute to host cell invasion because these differentiated cells can invade urothelial cells faster and more prominently than vegetative cells ${ }^{5}$. Swimming motility is also thought to contribute to dissemination within the urinary tract, in particular regarding the ascension from the bladder to the kidneys and spread between kidneys. Flagella clearly contribute to $P$. mirabilis pathogenesis ${ }^{6}$.

Three potential toxins have been characterized for their important role in virulence. These are hemolysin, Proteus toxic agglutinin (pta), and ZapA metalloprotease. Hemolysin could play a role in the spread of infection into the kidneys and the initiation of acute pyelonephritis. pta is an auto-transporter that performs serine protease activity on the surface of bacteria ${ }^{7,8}$. The pta protein contributes to the colonization of the bladder and kidney. In vitro and in vivo UTI studies have demonstrated the additive effects of hpmA and pta, particularly with respect to cystitis and possibly interstitial nephritis ${ }^{9-11}$. ZapA Metalloprotease is capable of cleaving $\operatorname{IgA}, \operatorname{IgG}$, antimicrobial peptides hBD1 and LL-37, complement proteins C1q and C3, fibronectin, actin, collagen, laminin, casein, and gelatin. Zap protease and hemolysin may cause swarm cells to be more cytotoxic to the host urothelium ${ }^{5}$.

Bacteria utilize quorum sensing to control biofilm, toxin, exopolysaccharide, virulence factor production, and motility, all of which are necessary for the effective foundation pathogenic relation with eukaryotic hosts ${ }^{11}$. P. mirbilis fimbriae contribute to infection in the kidney and bladder, although the receptors involved have not been recognized yet. The attachment of fimbriae to renal cells to initiate pyelonephritis and the critical role of fimbriae in cystitis have been demonstrated in previous studies ${ }^{12}$. Mannose-resistant Proteus-like fimbriae $(\mathrm{MR} / \mathrm{P})$ are expressed in the urinary tract and contribute to virulence. Direct observations of $P$. mirabilis in the bladder, urine, and kidneys of mice revealed MR/P fimbriation in all parts of the urinary tract ${ }^{13}$. However, up to $85 \%$ of bacteria do not express MR/P in the kidneys ${ }^{14}$. Phase variation of the mrp promoter orientation may contribute to the evasion of host defences.

The formation of biofilms on catheter surfaces, including urinary catheters, is considered a notorious problem. The formation of biofilm depends on MR/P fimbriae. The formation of biofilm in catheters and uri- nary tissue is associated with P. mirabilis catheterization. Crystalline biofilms are formed by depositing struvite and apatite minerals among the colonized surfaces in the presence of urine. The urease activity of bacteria increases the local $\mathrm{pH}$, eventually leading to the deposition of minerals ${ }^{15}$. Crystalline biofilms can obstruct catheters, and, therefore, P. mirabilis is especially problematic for patients with indwelling urinary catheters.

P. mirabilis is isolated between $1-10 \%$ of all UTIs, without considering the geographic location of the study, the types of samples collected. In recent studies, this species was found in 5-20\% of cases and had a mortality rate of as high as $50 \%$ in geriatric patients ${ }^{7,16}$.

P. mirabilis has a broad range of virulence factors, each of which plays a vital role in UTIs. These factors are associated with the relationship between bacteria and surfaces, invasion, damage to host tissues escaping from the host's immune system, and iron absorption ${ }^{17}$.

There is a lack of research considering the epidemiology, and prevalence of serogroups, the frequency of virulence factors, and the characteristics of antibiotic resistance regarding $P$. mirabilis in Iran. This study was designed to characterize virulence genes and antimicrobial resistance patterns in P. mirabilis isolated from patients with urinary infections who were admitted to tertiary hospitals in Iran.

\section{Methods}

In our study, a total of 100 isolates of P.mirabilis causing UTIs were isolated from patients and outpatients in hospitals affiliated to University of Medical Sciences of Tehran, Iran from August 2016 to August 2018. The selection of the subjects in this study was in accordance with CDC guideline. The recruited patients met the following criteria: temperature more than $38^{\circ} \mathrm{C}$ (fever) need to severe urinary excretion, with frequent urination, Dysuria, incomplete bladder emptying, Supra-pubic and flank pain, presence of leukocytes or blood in the urine and finally, a positive culture with more than $105 \geq \mathrm{CFU} / \mathrm{ml}$ colonies. Information on patients including the types of UTIs, relapses, age; sex and etc. were collected with the patient's consent.

\section{Identification and preservation of $P$. mirabilis strains} Identification of the strains was performed using the API tests: API 20E/ID32E (BioMérieux), according to the manufacturers' recommendations. Strains were stored in a brain heart infusion with $20 \%$ glycerol at $-70{ }^{\circ} \mathrm{C}$. 


\section{Antimicrobial susceptibility testing}

The antimicrobial susceptibility testing was performed using the disk diffusion method to amoxicillin with clavulonic acid, piperacillin with tazobactam, cefotaxime, cetriaxone, cefepime, amikacin, gentamicin, ciprofloxacin, nalidicxic acid, trimethoprim-sulfamethoxazole(SXT), imipenem, and meropenem according to the Clinical and Laboratory Standards Institute (CLSI 2017) guidance ${ }^{18,19}$.

\section{Evaluation of biofilm Formation}

The biofilm formation of the P. mirabilis strains was examined using the modified method described by Kwiecinska-Piróg et al. A 0.1\% (TTC) 2, 3, 5-Triphenyl-tetrazolium chloride solution with modifications was applied. After a 24 -hour incubation at $37^{\circ} \mathrm{C}$, planktonic, and non-adsorbed cells were washed from the wells. The wells were washed three times with $600 \mu \mathrm{L}$ sterile distilled water (DDW). Then, $100 \mu \mathrm{L}$ of tryptic soy medium (TSB, Becton Dickinson) and $100 \mu \mathrm{L}$ of sterile $0.1 \%$ TTC solution were added to each well and incubated for two hours at $37{ }^{\circ} \mathrm{C}$ temperature. The contents of the wells were removed and washed again with DDW. The formazan was suspended in $200 \mu \mathrm{L}$ methanol and added to each well. The contents of the wells were moved to sterile microtiter plates. The absorbance was counted using a BIO-TEK spectrophotometer at $470 \mathrm{~nm}$. The results were interpreted in accordance with the criteria described in the previous publication ${ }^{20}$, 21.

\section{Molecular detection of virulence genes}

Genomic DNA was extracted by DNA extraction kit (Roche, Switzerland) and stored at $-20{ }^{\circ} \mathrm{C}$. PCR approach was used to detect the presence of virulence genes including (mrpH, ucaA, hpmA, zapA, ptaA, ureG, pmfA, fliC, mrpA). The primers of this study were specifically designed and synthesized by Gene Fannavaran
Company (Iran). The primer sequences, their annealing temperatures and product sizes are given in Table 1. The PCR was performed in Eppendorf Thermal Cyclers (Eppendorf, USA) ${ }^{22}$.

\section{Statistical analysis}

Data were analyzed using statistical analysis software, SPSS version 24.0 (IBM, Chicago, USA). Categorical data were analyzed using chi-square or Fisher's exact tests. P-value $<0.05$ was considered significant.

\section{Results}

\section{Isolation of $P$. mirabilis}

A total of 100 specimens representing patients clinically were diagnosed as UTI patients distributed as 40 Isolates from males and 60 Isolates from females. 35 $(35 \%)$ strains were isolated from urine collected from catheterized, while $65(65 \%)$ strains were isolated from the urine of non-catheterized patients.

\section{Antimicrobial Susceptibility Profile}

The results of the antibiotic susceptibility testing are shown in Figure 1. Of the 100 P. mirabilis strains isolated from UTI, $3(3 \%)$ were susceptible to all antimicrobials tested, while $16(16 \%)$ were observed to be unsusceptible to more than three antimicrobial families and were identified as multidrug-resistant (MDR). Twenty isolates were resistant to imipenem and 18 isolates were resistant to meropenem. $77(77 \%)$ isolates demonstrated resistance to the extended spectrum $\beta$-lactam antibiotics in the disk diffusion test. Nalidixic acid SXT had the highest resistance. The antimicrobial susceptibility profile of the P. mirabilis isolates revealed that $72 \%$ and $71 \%$ were susceptible to imipenem, meropenem, $82 \%$ to amikacin, $81 \%$ to ciprofloxacin, and $73 \%$ to ceftriaxone. Antimicrobial resistance was observed against the SXT (97\%), nalidixic acid (93\%) and amoxicillin (62\%) antibiotics.

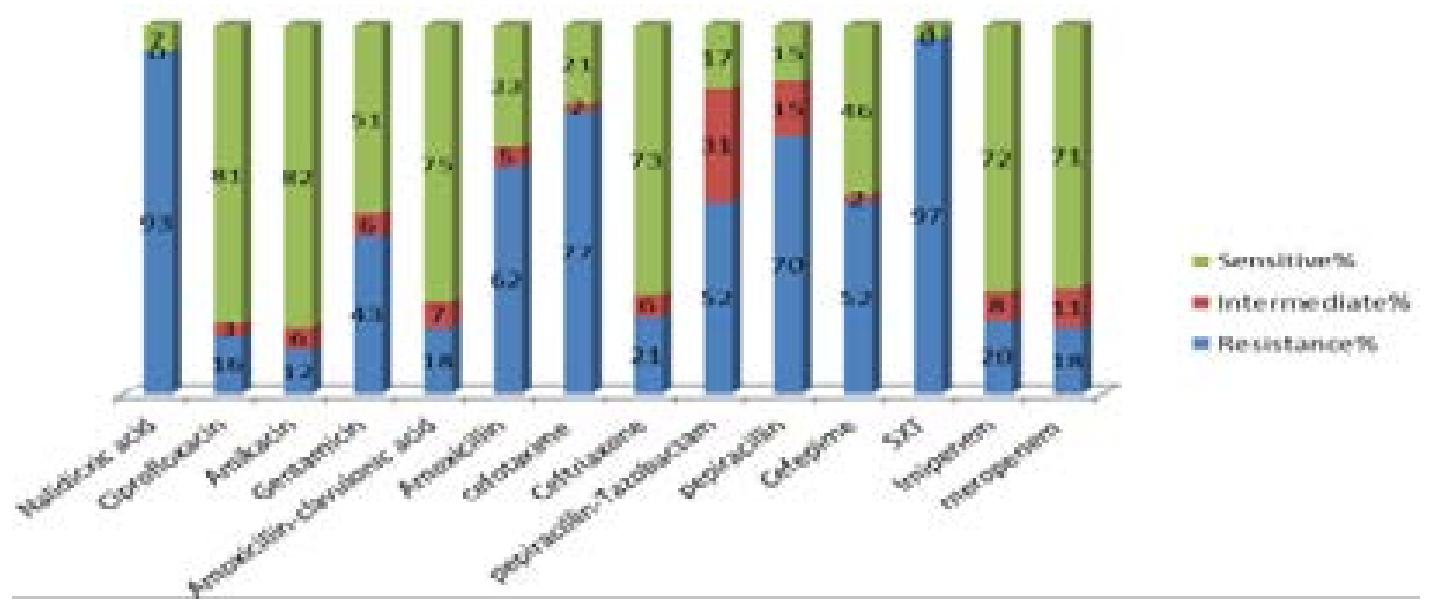

Fig 1. The susceptibly pattern of 100 Proteus mirabilis isolates to 14 antimicrobial agents. 
isolates to 14 antimicrobial agents.

Circulation of Virulence Genes in P. mirabilis isolated from UTI

Among the nine virulent genes, mrpA was detected at a ratio of $90 \%(90 / 100)$, while $\mathrm{mrpH}$, ptaA, ureG and
pmfA exhibited a similar percentage; 100\% (100/100) was observed in P. mirabilis. Moreover, among the nine virulent genes, ucaA was detected at a ratio of $91 \%$ (91/100), while zapA and fliC exhibited similar percentages; $95 \%$ of zapA, and $97 \%$ of fliC were observed in P. mirabilis (Fig 2).

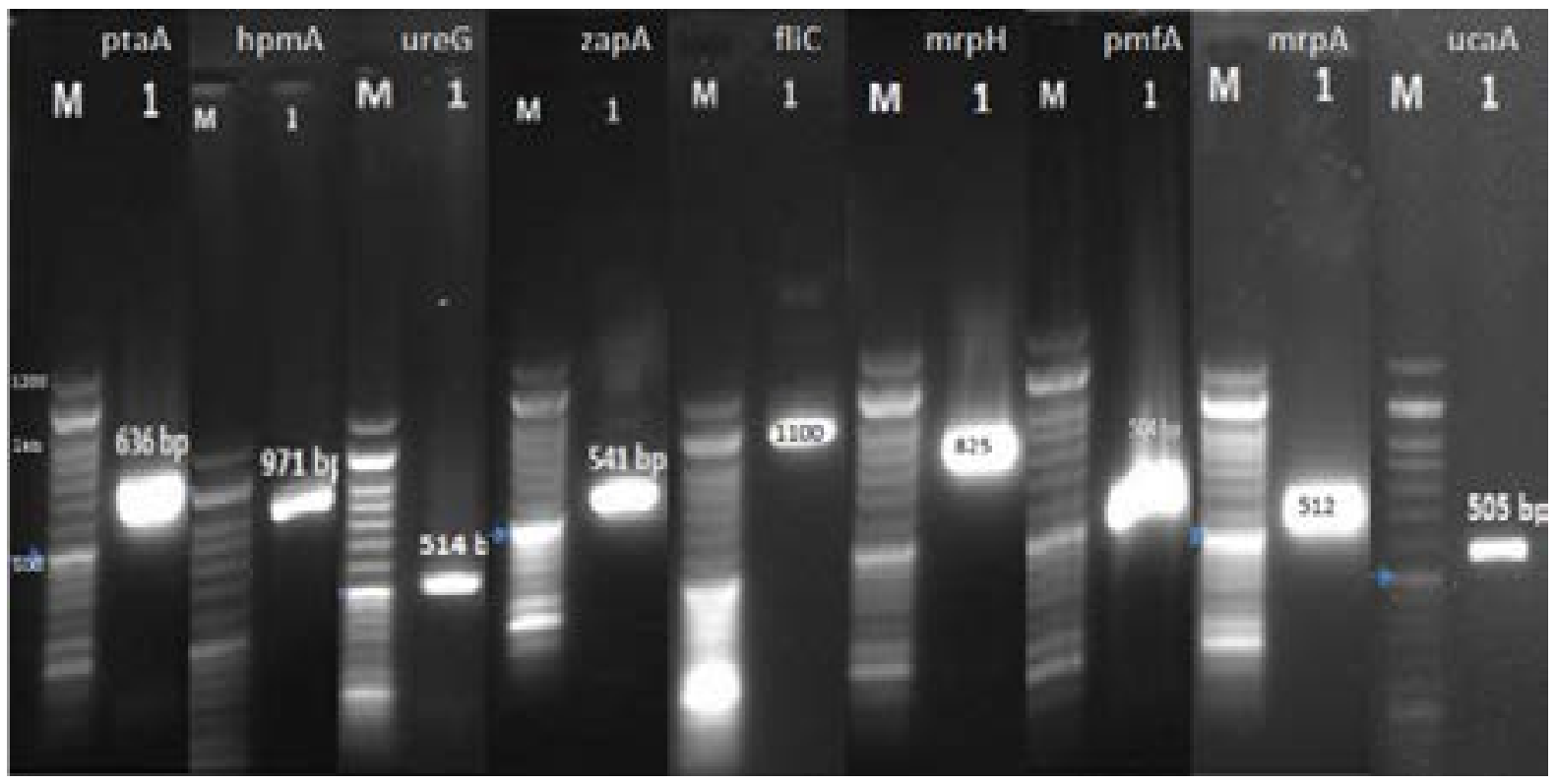

Fig 2. An electrophoresed gel showing PCR products. The left most lane represents a DNA ladder with fragments at $100 \mathrm{bp}$ intervals.

Comparison of the urine-derived P. mirabilis strains; the ability to form a biofilm with respect to catheterization of the patients.

The potency to form biofilm by $P$. mirabilis strains isolated from non-catheterized and catheterized patients showed significant differences. A higher percentage $(83.3 \%)$ of the strains in this case was classified as weak biofilm producers among non-catheters. A higher percentage $(80 \%)$ of the strains in this case was classified as strong biofilm producers among catheters patients (Table 2). 
Table 1: list of primers which were used in this study

\begin{tabular}{|c|c|c|c|c|c|}
\hline & primer sequence ( 5 & $\begin{array}{l}\text { Target } \\
\text { genes }\end{array}$ & Size(bp) & $\begin{array}{l}\text { Annealing } \\
\text { Tem }\end{array}$ & Reference \\
\hline 1 & $\begin{array}{l}\text { F: TTC TTA CTG ATA AGA CAT TG } \\
\text { R: ATT TCA GGA AAC AAA AGA TG }\end{array}$ & $\operatorname{mrpA}$ & 512 & 56 & This study \\
\hline 2 & $\begin{array}{l}\text { F:CTGCGGCTTTAGTATTTGGT } \\
\text { R:TAACGGCTTGGAATTCACCT }\end{array}$ & $p m f A$ & 504 & 47 & This study \\
\hline 3 & $\begin{array}{l}\text { F:CATGCCATGAAAAGAAAAGTTATAGC } \\
\text { R:CCCAAGCTTCTCATAGGCAATGGTGTAAT }\end{array}$ & ucaA & 505 & 56 & This study \\
\hline 4 & $\begin{array}{l}\text { F:AGAATATAATCAACCACTGCGTA } \\
\text { R:CATTTTGGCTGTATCCGCTTC }\end{array}$ & ureG & 514 & 47 & This study \\
\hline 5 & $\begin{array}{l}\text { F:CAATTTCAGCACCTAATAACCC } \\
\text { R:TGCTTAATCAAGGAGCCGAT }\end{array}$ & $\operatorname{ptaA}$ & 636 & 47 & This study \\
\hline 6 & $\begin{array}{l}\text { F:ATAGTCACGCCAAATAACGAA } \\
\text { R:TATTTCCACGAGTAGAACCAG }\end{array}$ & hmpA & 971 & 47 & This study \\
\hline 7 & $\begin{array}{l}\text { F:TATCGCAGAACTGATCACTCG } \\
\text { R:ATCTGGCTCTTTGTTAGCTTG }\end{array}$ & zapA & 541 & 47 & This study \\
\hline 8 & $\begin{array}{l}\text { F:CATGCCATGGCGATGGCACAAGTCATTAAT } \\
\text { R:CCGCTCGAGACGTAACAGAGACAGAACA }\end{array}$ & fliC & 1100 & 55 & This study \\
\hline 9 & $\begin{array}{l}\text { F:CATGCCATGGCCATGTTTATATTTAAACGA } \\
\text { TT } \\
\text { R:CCCAAGCTTAGGCATGGTTAAAATAATTG }\end{array}$ & $m r p H$ & 828 & 55 & This study \\
\hline
\end{tabular}


Table 2: Comparison of the urine-derived P. mirabilis strains ability to form

biofilm with respect to catheterization of the patients

\begin{tabular}{|ll|l|l|l|l|l|}
\hline \multicolumn{2}{|l|}{ Biofilm } & Lack & Weak & Moderate & Strong & \\
Patients & N (\%) & 50 & 12 & 13 & 25 & \multirow{2}{*}{ P value } \\
\cline { 1 - 5 } Catheterized $35(35 \%)$ & $5(10 \%)$ & $2(16.7 \%)$ & $8(61.5 \%)$ & $20(80 \%)$ & \\
\cline { 1 - 5 } Non- Catheterized 65(65\%) & $45(90 \%)$ & $10(83.3 \%)$ & $5(38.5 \%)$ & $5(20 \%)$ & \multirow{2}{*}{0.00001} \\
\hline
\end{tabular}

\section{Discussions}

The emergence of MDR strains that are resistant to most of the tested antimicrobials agents might be because of the use of easily available prescription and non-prescription drugs before the urine culture results were obtained. The widespread use (and often misuse) of antimicrobial drugs has led to a general rise in the emergence of resistant bacteria.

The results also showed that $62(62 \%)$ and $70(70 \%)$ isolates were resistant to amoxicillin and piperacillin, respectively. These surveys are consistent with studies that showed that Proteus isolates were unsusceptible to ampicillin and piperacillin and that reported that ampicillin has no effect on any of the isolates of UTIs ${ }^{22}$.

A major virulence factor of these bacteria is their ability to create a biofilm. Biofilm protects bacteria from the host's immune system response and restricts the penetration of antibiotics and antibodies ${ }^{23}$.

The typical effects of the biofilm-trapped bacteria are an almost-1000-fold increased resistance to most antimicrobials when compared to planktonic bacteria. The biofilm formed on the abiotic surfaces is a major cause of $65 \%$ of nosocomial infections ${ }^{20}$.

P. mirabilis showed the potential to create biofilm in various environments, including on abiotic (catheter) and biological surfaces ${ }^{24}$. It might cause urine obstruction in the bladder, bacteriuria recurrent, fever, sepsis, and shock. ${ }^{25}$

In the present study, $90 \%$ of strains had the mrpA gene, inclding $70 \%$ in cystitis and $30 \%$ in pyelonephritis. Sosa et al. studied clinical and non-clinical strains and found that several virulence factors (e.g., swarming, urease) are associated with the uropathogenic $P$. mirabilis. Hemolysin production and various instances of fimbrial gene expression were analyzed; the data showed that all the strains have mrpA, pmfA, and ucaA genes ${ }^{26}$.
In another study on urinary $P$. mirabilis strains in which virulence factors such as urease, protease, hemolysin, and the ability of swarming/span $>$ were evaluated and measured, all studied strains had ureC and zapA genes ${ }^{27}$. The prevalence rates of the genes related to the virulence factors based on the multiplex PCR method were ureA (96.7\%), ureC (100\%), hpmA (100\%), zapA $(100 \%)$, and flaA $(86.7 \%)^{27}$. The majority of the isolated strains in the current study contained fliC, zapA, hpmA, and ucaA genes; similar results were obtained in other studies ${ }^{28}$.

The zapA and mrpA genes are mainly important for the adherence were identified in $95 \%$ and $90 \%$ isolates, respectively. However, in the study by Holling et al., the frequencies of both genes in P. mirabilis was $73.3 \%$, which contrasts with other previous studies, which reported a frequency of $30 \%{ }^{29,30}$. In our study, the most important gene was mrpA per its role in several virulence factors ${ }^{30}$. The genes involved in biofilm formation, such as pmfA, ucaA, mrpH, mrpA, and fliC, were found in the majority of the strains, possibly resulting in high-intensity biofilm formation and thus increasing antibiotic resistance among the strains ${ }^{20}$.

P. mirabilis with controlling antibodies and antimicrobial peptides can lead to UTIs. To do this, P. mirabilis encodes a protease that is capable of mediating the degradation of the $\beta$-defensin- 1 and LL-37 that are present in the urinary tract ${ }^{20,30}$. The ZapA-mediated degradation of $\beta$-defensin- 1 and LL-37 decreases their antimicrobial activity ${ }^{31}$.

The formation of biofilm, evaluation of hemagglutination, and measurement of virulence markers were studied in the urinary $P$. mirabilis strains of patients with UTIs in Italy (2006). The results showed that all the strains contained mrpA and $\mathrm{mrpH}$ genes ${ }^{32}$. 
Biofilms, which are adherent microbial communities, are a notorious problem on catheter surfaces (including urinary catheters) and contribute to disease. Because catheterization is a major risk factor for $P$. mirabilis UTIs, biofilms within catheters and urinary tissues must be considered. MrpA is the main structural subunit of $\mathrm{MR} / \mathrm{P}$, and its expression is increased when oxygen is limited, which is logical for a virulence factor, given the reduced oxygen availability in the bladder ${ }^{11}$.

Experiments conducted by Tsai et al. ${ }^{25}$ suggest that $\mathrm{MR} / \mathrm{P}$ fimbriae dictate the localization of bacteria in the bladder and contribute to biofilm formation, a process that is essential for the establishment of catheter-associated UTIs. MR/K fimbriae can cause the adhesion of bacteria to catheter surfaces and the permanence of catheter-related bacteriuria.

\section{Conclusion}

The $P$. mirabilis strains isolated in the current study are accompanied by several virulence factors, including adherence factors, hemolysin, urease, and swarming activity. The presence of important virulence factors was further validated using a PCR approach.

\section{Abbreviations \\ UTIs: Urinary Tract Infections; PCR: Polymerase Chain Reaction; ICU: Intensive Care Unit; CLSI: Clinical and Laboratory Standards Institute \\ Declarations \\ Ethics approval and consent to participate \\ Consent for participation in research was obtained from all participants. This study was conducted under the approval of the Rasool-e-Akram hospital.}

\section{Consent to publication}

Not applicable.

\section{Availability of data and materials}

The datasets used and/or analyzed during the current study are available from the corresponding author on reasonable request.

\section{Competing interests}

Portions of the authors' time were supported by a grant from the Research Center of Pediatric Infectious Diseases.

\section{Conflict of Interests}

The authors report no potential conflict of interests.

\section{Funding}

This project was supported financially by Research Center of Pediatric Infectious Diseases, Rasool Akram Hospital, Iran University of Medical Sciences, Tehran, Iran.

\section{Acknowledgments}

This research was financially supported by the Iran University with funds from the maintenance of the research potential of the Rasool -e- Akram Teaching Hospital.

\section{References}

1. Getliffe, K. and T. Newton, Catheter-associated urinary tract infection in primary and community health care. Age and Ageing, 2006. 35(5): p. 477-481.

2. Jacobsen, S., et al., Complicated catheter-associated urinary tract infections due to Escherichia coli and Proteus mirabilis. Clinical Microbiology Reviews, 2008. 21(1): p. 26-59.

3. Prywer, J. and M. Olszynski, Bacterially induced formation of infectious urinary stones: recent developments and future challenges. Current Medicinal Chemistry, 2017. 24(3): p. 292-311.

4. Cusick, K., et al., Perturbation of FliL interferes with Proteus mirabilis swarmer cell gene expression and differentiation. Journal of Bacteriology, 2012. 194(2): p. 437447.

5. Alamuri, P., et al., Adhesion, invasion, and agglutination mediated by two trimeric autotransporters in the human uropathogen Proteus mirabilis. Infection and Immunity, 2010. 78(11): p. 4882-4894.

6. D'Alessandro, B., et al., Proteomic analysis of Proteus mirabilis outer membrane proteins reveals differential expression in vivo vs. in vitro conditions. FEMS Immunology \& Medical Microbiology, 2011. 63(2): p. 174182.

7. Adams-Sapper, S., et al., Globally dispersed mobile drug-resistance genes in Gram-negative bacterial isolates from patients with bloodstream infections in a US urban general hospital. Journal of Medical Microbiology, 2012. 61(Pt 7): p. 968.

8. Sader, H.S., R.K. Flamm, and R.N. Jones, Frequency of occurrence and antimicrobial susceptibility of Gram-negative bacteremia isolates in patients with urinary tract infection: results from United States and European hospitals (2009-2011). Journal of Chemotherapy, 2014. 26(3): p. 133-138.

9. Alamuri, P., et al., Vaccination with proteus toxic agglutinin, a hemolysin-independent cytotoxin in vivo, protects against Proteus mirabilis urinary tract infection. Infection and Immunity, 2009. 77(2): p. 632-641. 
10. Alamuri, P. and H.L. Mobley, A novel autotransporter of uropathogenic Proteus mirabilis is both a cytotoxin and an agglutinin. Molecular Microbiology, 2008. 68(4): p. 997-1017.

11. Belas, R., J. Manos, and R. Suvanasuthi, Proteus mirabilis ZapA metalloprotease degrades a broad spectrum of substrates, including antimicrobial peptides. Infection and Immunity, 2004. 72(9): p. 5159-5167.

12. Scavone, P., et al., Role of Proteus mirabilis MR/P fimbriae and flagella in adhesion, cytotoxicity and genotoxicity induction in T24 and Vero cells. Pathogens and Disease, 2015. 73(4).

13. Zisman, A.L., Effectiveness of treatment modalities on kidney stone recurrence. Clinical Journal of the American Society of Nephrology, 2017. 12(10): p. 1699-1708.

14. Nicholson, E.B., et al., Proteus mirabilis urease: transcriptional regulation by UreR. Journal of Bacteriology, 1993. 175(2): p. 465-473.

15. Pellegrino, R., et al., Proteus mirabilis uroepithelial cell adhesin (UCA) fimbria plays a role in the colonization of the urinary tract. Pathogens and Disease, 2013. 67(2): p. 104-107.

16. Lubart, E., et al., Bacteremia in a multilevel geriatric hospital. Journal of the American Medical Directors Association, 2011. 12(3): p. 204-207.

17. O'Hara, C.M., F.W. Brenner, and J.M. Miller, Classification, identification, and clinical significance of Proteus, Providencia, and Morganella. Clinical Microbiology Reviews, 2000. 13(4): p. 534-546.

18. Wayne, P.A., Clinical and laboratory standards institute. Performance standards for antimicrobial susceptibility testing. 2017.

19. Badamchi, A., et al., Phenotypic and genotypic characterization of antibiotic resistance in Klebsiella pneumoniae isolated from patients admitted to a third-level hospital in Tehran, Iran.

20. Kwiecinska-Piróg, J., et al., Proteus mirabilis biofilm-Qualitative and quantitative colorimetric methods-based evaluation. Brazilian Journal of Microbiology, 2014. 45(4): p. 1423-1431.
21. Xu, Z., et al., Crystal violet and XT'T assays on Staphylococcus aureus biofilm quantification. Current Microbiology, 2016. 73(4): p. 474-482.

22. Pathirana, H., et al., Comparison of virulence genes in Proteus species isolated from human and pet turtle. Iranian Journal of Veterinary Research, 2018. 19(1): p. 48.

23. Hengge, R., Targeting bacterial biofilms by the green tea polyphenol EGCG. Molecules, 2019. 24(13): p. 2403. 24. Jacobsen, S.M. and M.E. Shirtliff, Proteus mirabilis biofilms and catheter-associated urinary tract infections. Virulence, 2011. 2(5): p. 460-465.

25. Tsai, Y.-L., et al., cAMP receptor protein regulates mouse colonization, motility, fimbria-mediated adhesion, and stress tolerance in uropathogenic Proteus mirabilis. Scientific Reports, 2017. 7(1): p. 7282.

26. Holling, N., et al., Elucidating the genetic basis of crystalline biofilm formation in Proteus mirabilis. Infection and Immunity, 2014. 82(4): p. 1616-1626.

27. Rocha, S.P., et al., Aggregative adherence of uropathogenic Proteus mirabilis to cultured epithelial cells. FEMS Immunology \& Medical Microbiology, 2007. 51(2): p. 319-326.

28. Manikandan, S., et al., Antimicrobial susceptibility pattern of urinary tract infection causing human pathogenic bacteria. Asian Journal of Medical Sciences, 2011. 3(2): p. 56-60.

29. Abbas, K.F., et al., Molecular detection of some virulence genes in Proteus mirabilis isolated from Hillaprovince. International Journal of Research Studies in Biosciences (IJRSB, 2015. 3: p. 85-89.

30. Chromek, M., et al., The antimicrobial peptide cathelicidin protects the urinary tract against invasive bacterial infection. Nature Medicine, 2006. 12(6): p. 636. 31. Sosa, V., G. Schlapp, and P. Zunino, Proteus mirabilis isolates of different origins do not show correlation with virulence attributes and can colonize the urinary tract of mice. Microbiology, 2006. 152(7): p. 2149-2157.

32. Lane, M.C., et al., Oxygen-limiting conditions enrich for fimbriate cells of uropathogenic Proteus mirabilis and Escherichia coli. Journal of Bacteriology, 2009. 191(5): p. 1382-1392. 